\title{
Stringent and efficient assessment of Boson-Sampling devices
}

\author{
Malte C. Tichy, ${ }^{1}$ Klaus Mayer, ${ }^{2}$ Andreas Buchleitner, ${ }^{2}$ and Klaus Mølmer ${ }^{1}$ \\ ${ }^{1}$ Department of Physics and Astronomy, Aarhus University, DK-8000 Aarhus, Denmark \\ ${ }^{2}$ Physikalisches Institut, Albert-Ludwigs-Universität Freiburg, D-79104 Freiburg, Germany
}

(Dated: June 8, 2018)

\begin{abstract}
Boson-Sampling holds the potential to experimentally falsify the Extended Church Turing thesis. The computational hardness of Boson-Sampling, however, complicates the certification that an experimental device yields correct results in the regime in which it outmatches classical computers. To certify a boson-sampler, one needs to verify quantum predictions and rule out models that yield these predictions without true many-boson interference. We show that a semiclassical model for many-boson propagation reproduces coarse-grained observables that were proposed as witnesses of Boson-Sampling. A test based on Fourier matrices is demonstrated to falsify physically plausible alternatives to coherent many-boson propagation.
\end{abstract}

Introduction - According to the Extended Church Turing thesis (ECT), any efficient computation performed by a physical device can also be performed efficiently (with polynomial overhead) by a classical computer [1. Since the advent of quantum computation - especially since the formulation of Shor's factoring algorithm [2, 3, - the ECT has been under attack, since quantum computers are believed to outperform classical devices. Nevertheless, the available empirical evidence is insufficient to dismiss the ECT as a central dogma of computer science, and a functional universal quantum computer is not likely to be constructed in the foreseeable future.

A more approachable challenge to the ECT is provided by Boson-Sampling [4, which is harder than factoring, while it can be solved efficiently by a quantum device of modest capabilities: Only the coherent propagation of many identical bosons through a multimode setup is required. Experimental boson-samplers with three photons match the theoretically expected particle distribution [58. Scaling to larger photon numbers is equally challenging [9 11] as conceptually indispensable, and may be alleviated by alternative formulations of the problem that keep its computational hardness [12, 13].

The certification of an alleged boson-sampler in the regime of many particles is decisive for a serious and well-founded attack on the ECT. Under the assumption that quantum physics correctly describes the propagation of arbitrarily many bosons, no certification issue arises at all, and no traditionally trained physicist will question the implications of Boson-Sampling. But in a crossdisciplinary context that encompasses computer science, mathematics and physics, the validity of quantum mechanics for truly many interfering particles must be underpinned by unambiguous empirical evidence.

The very hardness of Boson-Sampling makes such desirable certification a dilemma: On the one hand, it quickly becomes unfeasible to compute the full BosonSampling distribution classically, because the computational expenses for a single transition probability as well as the total number of events diverge exponentially in the number of bosons $n$. On the other hand, one may measure efficiently predictable observables such as statistical bosonic signatures, but such strategy leaves room for alternative models that explain the observed behavior without the complex interference of many bosons.

The persuasiveness of any certification protocol therefore hinges on how convincingly it establishes the quantum prediction for many bosons while ruling out alternative models. Several efficient certification protocols have been devised [14-17]; in particular those recently put forward in Ref. [16, 17, discriminate the bosonic output against the classical behavior of distinguishable particles. Here, we show that certificates based on bosonic bunching are nevertheless not stringent, because they (erroneously) qualify the output of the efficient and physically plausible semi-classical mean-field sampler (described below) as a functional boson-sampler. We devise an efficient and more stringent test based on highly symmetric sampling matrices, which can conclusively rule out the meanfield sampler and leaves no room for physically plausible models that pass the test without invoking the granular quantum interference of $n$ bosons. By assessing the gradual failure of the test due to inaccuracies, we establish the experimental requirements for a certifiable device.

Sampling and certification - Boson-Sampling consists in simulating output events of $n$ bosons prepared in the $n$ different input ports $\vec{j}=\left(j_{1}, \ldots, j_{n}\right)$ out of the $m \gg n$ modes of a scattering setup chosen randomly according to the Haar measure on $m \times m$ unitary matrices $U$. That is, one draws output events $\vec{k}=\left(k_{1}, \ldots, k_{n}\right)$ with probability $P_{\mathrm{B}}(\vec{j}, \vec{k} ; U)$, which corresponds to the permanent of the sub-matrix of $U$ that contains the rows and columns matching the occupied input and output modes,

$$
P_{\mathrm{B}}(\vec{j}, \vec{k} ; U)=|\operatorname{permanent}(M)|^{2}, \quad M_{l, q}=U_{j_{l}, k_{q}},(1)
$$

where additional combinatorial factors arise for multiply occupied output modes. The permanent eludes polynomial algorithms, which is inherited by the above sampling problem: Physically speaking, the interference of $n$ ! many-particle paths [18] governs each event [see Fig. 1(d)]. An efficient classical algorithm for Boson- 
Sampling would imply extremely surprising consequences in computational complexity theory [4.

To certify a boson-sampler, one needs to verify predictions following from Eq. (1) and rule out models that yield these predictions without true many-boson interference 19. A certification protocol needs to be discarded if it accepts a series of events produced by a fraudulent device. Relevant fraudulent devices are those based on a plausible physical mechanism that can be simulated efficiently on a classical computer.

The simplest way to efficiently sample from the space of events $\vec{k}$ is uniform sampling [Fig. 11(a)], for which each event is assigned the same probability [19, and no information on the matrix $U$ or the initial state $\vec{j}$ is exploited. When $U$ and $\vec{j}$ are provided, one can distinguish BosonSampling from a uniform sampler via, e.g., the average number of particles in each output mode [14, 15, 20, which can be computed for the boson-sampler without evaluating any permanent,

$$
\left\langle\hat{n}_{k}\right\rangle=\sum_{l=1}^{n}\left|U_{j_{l}, k}\right|^{2} .
$$

Such single-particle observables reflect certain properties of the matrix $U$, but they are insensitive to manyparticle interference 18, 20. Therefore, single-particle observables are also replicated by efficient classical sampling, which can be implemented physically with distinguishable particles: Events are constructed by choosing the output mode $k$ for each particle prepared in $j_{q}$ with probability $p_{j_{q}, k}=\left|U_{j_{q}, k}\right|^{2}$ [see Fig. 1(b)]. Singleparticle observables are therefore not sufficient to discriminate the output of a boson-sampler from a classical or a fermion-sampler [14, 15, 20].



FIG. 1: (color online) Sampling models. (a) Uniform sampler: The scattering matrix and the initial state are ignored. (b) Classical sampler: Distinguishable particles propagate independently without interference. (c) Mean-field sampler: Macroscopic interference and bosonic effects are incorporated. (d) Boson-Sampling requires the interference of all $n$ ! paths of the $n$-boson wavefunction.

To rule out the classical sampler, appropriate coincidence and correlation observables were proposed in [16. 17]: The probability $P_{1}$ to find an $n$-fold coincidence outcome (without any multiply populated mode) is significantly higher for distinguishable particles than for bosons due to the bunching tendency of the latter. Similarly, one can leave the space of random matrices and focus on structured multimode setups with certain features: Bosonic clouding [16] is the tendency for bosons to favor events with all particles in the same half of the output array of a continuous-time many-particle quantum walk. The two proposed observables, however, do not unambiguously verify the many-body coherence of the wavefunction, since the statistical tendency to multiply populate output states survives the deterioration of granular many-body interference: In the mean-field sampler, the Wigner function [21] of the multi-mode Fock-state is semi-classically approximated by the macroscopically populated single-particle states [see Fig. 1(c)]

$$
|\psi\rangle=\frac{1}{\sqrt{n}} \sum_{r=1}^{n} e^{i \theta_{r}}\left|\phi_{j_{r}}^{(\mathrm{in})}\right\rangle,
$$

where the phases $\theta_{r}$ are undefined 22 24. That is to say, the mean field forms a thin belt on the high-dimensional Bloch-sphere [21], which evolves into

$$
\hat{U}|\psi\rangle=\frac{1}{\sqrt{n}} \sum_{q=1}^{m}\left[\left|\phi_{q}^{\text {(out) }}\right\rangle\left(\sum_{r=1}^{n} e^{i \theta_{r}} U_{j_{r}, q}\right)\right],
$$

i.e. each particle occupies the output mode $1 \leq$ $q \leq m$ with probability $p_{q}^{\mathrm{mf}}=\left|\left\langle\phi_{q}^{\text {(out) }}|\hat{U}| \psi\right\rangle\right|^{2}=$ $\frac{1}{n}\left|\sum_{r=1}^{n} e^{i \theta_{r}} U_{j_{r}, q}\right|^{2}$ [23]. The ensemble average consists in sampling over random phases $\left\{\theta_{1} \ldots \theta_{n}\right\}$, each setting then leaves the particles (classically) correlated, since particles gather where $p_{q}^{\mathrm{mf}}$ is high.

The mean-field sampler is an efficiently evaluable and physically plausible model: It contains those aspects of many-boson dynamics that survive in the semi-classical limit, in which fragile many-boson quantum interference is lost, and it describes experiments with interfering Bose-Einstein condensates [25, 26]. It can be implemented alternatively by sequentially preparing $n$ particles in the same initial state (3).

The mean-field sampler yields the expected mean occupation (2) and, as shown in Fig. 2, it reproduces the coincidences $P_{1}$ and the clouding $C$ predicted for the bosonsampler, Eq. (1). This clearly dismisses these observables as witnesses of Boson-Sampling. All coarse-grained signatures that can be ascribed to bosonic statistics are reproduced by mean-field sampling and cannot validate the potential of a physical device to disprove the ECT. Similar to the request that stringent tests of entanglement rule out behavior borne by classical fields with random correlated phases [27, a certification protocol for boson-sampling must include tests which address properties that are not reproduced by the mean-field sampler.

Certification via Fourier matrices - A certification scheme which rules out plausible physical models that circumvent the evaluation of the permanent (1) needs to be based on efficiently predictable, fine-grained observables that are sensitive to granular $n$-body interference. Since event probabilities subjugated by $n$-body interference are 




(b)

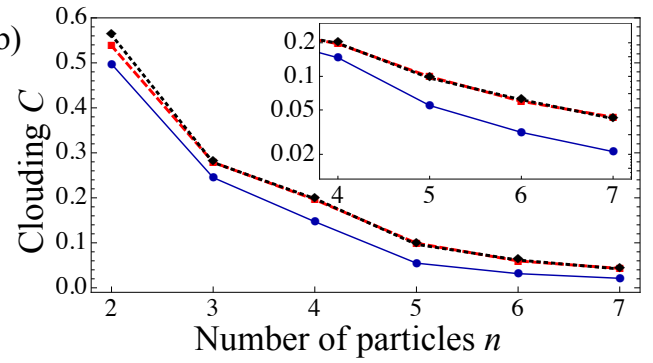

FIG. 2: (color online) Bunching and clouding in different sampling models. Classical distinguishable particles (blue circles) are compared to the mean-field sampler (red squares) and the boson-sampler (black diamonds). (a) Coincidence probability $P_{1}$ for 100 Haar-random unitary matrices of dimension $m=n^{2}$ with error-bars that represent one standard deviation (the mean-field sampler is hardly discernible from the boson-sampler). The lines are the combinatorially expected probabilities [16. (b) Clouding for a discrete-time quantum walk of 8 steps with $n$ particles starting in adjacent modes 20. 28. The probability $C$ that all particles be in the same half of the output array coincides for the mean-field and the boson-sampler.

hard to predict for unstructured random matrices, we leave the space of computationally hard sampling problems and choose a physically non-trivial, albeit efficiently predictable artificial instance of Boson-Sampling: The difficulty in the evaluation of the permanent in Eq. (1) in comparison to the benevolent determinant is due to the lack of symmetries. In order to alleviate the complexity, we choose a symmetric sampling matrix, the Fourier matrix of dimension $m=n^{p}$,

$$
U_{l, q}^{\text {Fou }}=\frac{1}{\sqrt{m}} \exp \left(i \frac{2 \pi l q}{m}\right)
$$

Cyclic symmetry is imposed on the initial state,

$$
\vec{j}_{\text {cyc }}=\left(1, n^{p-1}+1,2 n^{p-1}+1, \ldots,(n-1) n^{p-1}+1\right) \text {. }
$$

The cyclic symmetry remains intact during the scattering process, which is reflected by the occurring output events: Many events $\vec{k}$ are forbidden due to the suppression law for Fourier matrices [18, 24, 29]:

$$
\bmod \left(\sum_{l=1}^{n} k_{l}, n\right) \neq 0 \Rightarrow P_{\mathrm{B}}\left(\vec{j}_{\mathrm{cyc}}, \vec{k} ; U^{\text {Fou }}\right)=0,
$$

which generalizes the Hong-Ou-Mandel effect: For $\vec{j}=$ $(1,2)$, coincident output events with $\vec{k}=(1,2)$ lead to an odd sum in (7), two photons then never leave the beamsplitter in different modes [30].

The degree of violation of the suppression law is quantified by $\mathcal{V}=\mathcal{N}_{\text {forbidden }} / \mathcal{N}_{\text {runs }}$, the ratio of actually occurring events $\mathcal{N}_{\text {forbidden }}$ that violate Eq. $(7)$ to the total number of events $\mathcal{N}_{\text {runs }}$. An ideal boson-sampler features $\mathcal{V}=0$. The uniform, classical and mean-field samplers do not contain any mechanism to satisfy the suppression law, which leads to a considerable violation: Only a fraction $1 / n$ of a priori possible events can occur in an accurate Boson-Sampling experiment on the Fourier matrix (5) and the initial state (6), while most events are forbidden [29. For uniform, classical and mean-field sampling, one therefore observes $\mathcal{V} \approx(n-1) / n$ for large $n$ [see Fig. 3], and the probability that all events out of a sample of $R$ events accidentally fulfil the suppression law is $1 / n^{R}$. The Fourier matrix does not constitute a complex scenario, since forbidden events can be predicted efficiently: In practice, merely fractions of a second are required on a PC for $n \sim 10^{6}$. Notwithstanding, the observation of the suppression law in an experiment relies on granular $n$-particle interference: All $n$ ! many-particle amplitudes need to perfectly cancel, making the method stringent.

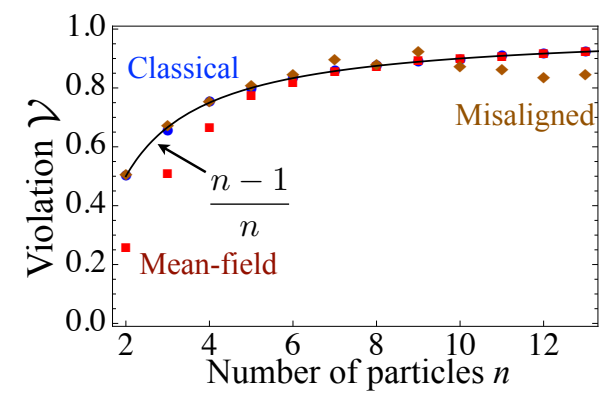

FIG. 3: (color online) Violation $\mathcal{V}$ of the suppression law $(7)$ by the classical (blue circles), mean-field (red squares) and misaligned (brown diamonds) sampler for $m=n^{2}$. The classical violation coincides with the ratio of suppressed events for the boson-sampler, $(n-1) / n$ (black solid line). For small particle numbers $n \lesssim 4$, the suppression law favors bunched states with many particles in few modes, which alleviates the violation by the mean-field sampler. For the misaligned data, we assume that one particle out of $n$ is distinguishable from the others, the total violation is inferred from computing the probability for up to 10000 distinct forbidden output states.

Can other models for many-body propagation fulfil the suppression law without true many-boson interference inherent to Eq. (1)? From the computational point of view, there are efficient fraudulent models: The output of a mean-field sampler can be checked against the suppression law, and forbidden events are blocked (alternatively, for odd $n$, bosonic forbidden events are also forbidden for efficiently simulatable fermions [18, although the latter do not bunch). From a physical perspective, however, there is no plausible mechanism that reads out the artifi- 
cial symmetries (5), (6) of the setup, establishes the suppression law (7), and implements an ad-hoc veto on the output states: The suppression of an event is a collective non-local property of the output state $\vec{k}$, which requires a physical mechanism that reigns over all particles in a concerted way. Hence, fulfilling the suppression law qualifies as the desired convincing "circumstantial evidence" [15] that an alleged boson-sampler is operational.

Deterioration due to inaccuracies - Our criterion based on the suppression law might appear too stringent in practice: Deviations from the ideal can be expected due to experimental inaccuracies, such as partial distinguishability of the bosons [31 34 and deviations of the scattering matrix $U$ from the Fourier matrix (5). The prediction of individual event probabilities in such scenario is unfeasible for many particles [31, but the large fraction $(n-1) / n$ of forbidden events allows us to efficiently estimate the violation $\mathcal{V}$, as shown in the following.

A state of partially distinguishable bosons reads 31]

$$
\left|\Psi^{\mathrm{ini}}\right\rangle=\prod_{r=1}^{n} \hat{a}_{j_{r}, t_{r}}^{\dagger}|\mathrm{vac}\rangle
$$

where the distinguishing degree of freedom $t_{r}$ accounts for, e.g., the mutual delay of injected photons. The states $\left|t_{1}\right\rangle, \ldots\left|t_{n}\right\rangle$ are Gram-Schmidt-orthogonalized to give the orthonormal basis $\left\{\left|t_{1}\right\rangle,\left|\tilde{t}_{2}\right\rangle \ldots\left|\tilde{t}_{n}\right\rangle\right\}$, which permits to expand $\left|\Psi^{\text {ini }}\right\rangle$ into $n$ ! orthogonal terms [31 33],

$$
\left|\Psi^{\mathrm{ini}}\right\rangle=\hat{a}_{j_{1}, t_{1}}^{\dagger} \sum_{d_{2}=1}^{2} \sum_{d_{3}=1}^{3} \cdots \sum_{d_{n}=1}^{n} \prod_{r=2}^{n} c_{r, d_{r}} \hat{a}_{j_{r}, \tilde{t}_{r}}^{\dagger}|\mathrm{vac}\rangle
$$

Each summand describes a different degree of interference capability: The term weighted by $c_{2,1} c_{3,1} \ldots c_{n, 1}$ with $d_{2 \ldots n}=1$ describes indistinguishable bosons that interfere perfectly and only give rise to non-forbidden events $(\mathcal{V}=0)$. The term with $d_{q}=q(2 \leq q \leq n)$ describes distinguishable particles, which induces $\mathcal{V} \approx$ $(n-1) / n$. Intermediate terms that describe neither fully distinguishable nor fully indistinguishable particles give rise to bosonic signatures such as bunching, but, in most cases, they do not fulfil the suppression law, and induce a violation of the order $(n-1) / n$. Even when merely one out of $n$ bosons is distinguishable, the suppression law is strongly violated [see brown diamonds in Fig. 3]. The total distinguishability-induced violation $\mathcal{V}_{\text {partial }}$ is therefore bounded by the weight of the perfectly indistinguishable term,

$$
\mathcal{V}_{\text {partial }} \lesssim \frac{n-1}{n}\left(1-\prod_{q=2}^{n}\left|c_{q, 1}\right|^{2}\right)
$$

Another experimental limitation is that the desired unitary transformation can be implemented only with limited accuracy. The probability for a forbidden transition $\vec{j} \rightarrow \vec{k}$ is not described by a submatrix $M$ of the Fourier matrix $U^{\text {Fou }}$ [see Eq. [5], but by a matrix $W$ with

$$
W_{l, q}=M_{l, q}\left(1+\delta_{l, q}\right) .
$$

By expanding the permanent of $W$ in powers of matrix elements of $\delta$ to the first order, we can estimate

$$
|\operatorname{permanent}(W)|^{2} \approx P_{\text {approx }}(\delta):=\frac{n \cdot n !}{m^{n}}\|\delta\|^{2},
$$

where $\|\delta\|:=\left\langle\left|\delta_{l, q}\right|\right\rangle_{l, q}$ is the average absolute value of matrix elements of $\delta$, which we assume to be much smaller than unity. For small deviations, the probability for non-forbidden events remains widely unaffected by $\delta$, and - circumventing the permanent - the violation can be estimated as

$$
\begin{aligned}
\mathcal{V}_{\mathrm{dev}} & \approx \frac{n-1}{n} N_{\text {events }} P_{\text {approx }}(\delta), \\
\text { for } & \underset{m}{\approx}=n^{2} \\
& \sqrt{e}(n-1)\|\delta\|^{2},
\end{aligned}
$$

where $N_{\text {events }}=\left(\begin{array}{c}m+n-1 \\ n\end{array}\right)$ is the total number of events. The estimate is confirmed numerically in Fig. 4 for $n=3,10, m=n^{2}$. Eqs. 13, (14) also formalize the



FIG. 4: (color online) Violation $\mathcal{V}$ for matrices described by Eq. 11, for $n=3,10$ and $m=n^{2}$. We compute the total probability of 200 randomly chosen forbidden events, for 400 different matrices $\delta$ for each value of the average deviation $\|\delta\|$. Error bars represent one standard deviation, the dashed red and solid blue lines show the estimate (14) for $n=10$ and $n=3$, respectively, which breaks down when $\|\delta\|$ is not smaller than $1 / n$. In order to observe the suppression law in the experiment, the violation needs to be significantly smaller than $(n-1) / n$ (compare to Fig. 3).

mild requirement on the accuracy of multimode scattering matrices that feature the suppression law. Since the two sources of deterioration are independent, the total expected violation can be estimated as $\mathcal{V}_{\text {total }} \approx$ $\mathcal{V}_{\text {partial }}+\mathcal{V}_{\text {dev }}$.

Outlook - The potential influence of a boson-sampler on the foundations of theoretical computer science is rather formidable, but so will be the requirements on convincing evidence for its proper functionality. Therefore, high exigency needs to be imposed on the falsification of alternative models for many-particle behavior. 
We showed that coarse-grained criteria based on bosonic bunching or clouding [16, 17] are insufficient, since they are reproduced by the semi-classical mean-field sampler [25, 26].

A functional boson-sampler will necessarily implement any unitary sampling matrix that the user wishes for to switch between different instances of the problem 35], and we can focus on the special instance described by (5), (6) to assess many-particle interference. The verification of (7) with three photons 36] and progress in integrated waveguide techniques 3739] feed the hope that the suppression law (7) will be observed in more complex setups in the near future. Within quantum mechanics, there are two sources of deviation from the ideal: Bosons can carry distinguishing degrees of freedom, and the setup might not precisely match the Fourier matrix. The deterioration induced by both effects can be estimated efficiently.

Following the spirit of the falsification of local realism [40, one may envisage a matrix similar to Eq. (5), but with hidden symmetries, such that events are forbidden according to an intricate rule that encodes the solution to a computationally hard problem. While sampling should therefore remain hard to perform, the output should be nevertheless easy to verify (in the language of computational complexity, the problem encoded by the matrix is in the complexity class NP). Such - admittedly speculative [4, 15] - instance of asymmetric complexity may offer an unquestionable computational criterion for the certification of boson-samplers, and promote such devices into powerful tools for algorithmic applications.

Acknowledgements. M.C.T. would like to thank Scott Aaronson, Christian Gogolin, Robert Keil and Karol Życzkowski for very helpful and clarifying discussions. M.C.T and K. Mølmer would like to thank the Villum foundation and the Danish Council for Independent Research.

[1] S. Aaronson, Quantum Computing since Democritus (Cambridge University Press, Cambridge, 2013).

[2] P. W. Shor, SIAM Rev. 41, 303 (1999).

[3] M. A. Nielsen and I. L. Chuang, Quantum computation and quantum information (Cambridge University Press, Cambridge, 2000).

[4] S. Aaronson and A. Arkhipov, STOC '11 43rd ann. ACM symp. Theo. Comp., 333 (2011).

[5] M. Tillmann, B. Dakić, R. Heilmann, S. Nolte, A. Szameit, and P. Walther, Nat. Photon. 7, 540 (2013).

[6] A. Crespi, R. Osellame, R. Ramponi, D. J. Brod, E. F. Galvao, N. Spagnolo, C. Vitelli, E. Maiorino, P. Mataloni, and F. Sciarrino, Nat. Photon. 7, 545 (2013).

[7] M. A. Broome, A. Fedrizzi, S. Rahimi-Keshari, J. Dove, S. Aaronson, T. C. Ralph, and A. G. White, Science 339, 794 (2013).

[8] J. B. Spring et al., Science 339, 798 (2013).

[9] P. P. Rohde, T. C. Ralph, Phys. Rev. A 85, 022332
(2012).

[10] V. S. Shchesnovich, Phys. Rev. A 89, 022333 (2014).

[11] A. Leverrier, R. García-Patrón Does Boson Sampling need Fault-Tolerance? arXiv:1309.4687, 2013.

[12] P. P. Rhode, K. R. Motes, and J. P. Dowling, Sampling generalized cat states with linear optics is probably hard, arXiv:1310.0297, 2013.

[13] A. P. Lund, A. Laing, S. Rahimi-Keshari, T. Rudolph, J. L. O'Brien, and T. C. Ralph, Boson sampling from gaussian states, arXiv:1305.4346v3, 2013.

[14] N. Spagnolo et al., Efficient experimental validation of photonic boson sampling against the uniform distribution, arXiv:1311.1622, 2013.

[15] S. Aaronson and A. Arkhipov, Bosonsampling is far from uniform, arXiv:1309.7460, 2013.

[16] J. Carolan et al., On the experimental verification of quantum complexity in linear optics, arXiv:1311.2913, 2013.

[17] J. Carolan et al., Verifying Quantum Complexity in Linear Optical Experiments, CLEO: 2014 (Opti. Soc. Am.) FM2A.7 (2014).

[18] M. C. Tichy, M. Tiersch, F. Mintert, and A. Buchleitner, New J. Phys 14, 093015 (2012).

[19] C. Gogolin, M. Kliesch, L. Aolita, and J. Eisert, Boson-sampling in the light of sample complexity, arXiv:1306.3995, 2013.

[20] K. Mayer, M. C. Tichy, F. Mintert, T. Konrad, and A. Buchleitner, Phys. Rev. A 83, 062307 (2011).

[21] J. P. Dowling, G. S. Agarwal, and W. P. Schleich, Phys. Rev. A 49, 4101 (1994).

[22] M. Chuchem, K. Smith-Mannschott, M. Hiller, T. Kottos, A. Vardi, and D. Cohen, Phys. Rev. A 82, 053617 (2010).

[23] W. J. Mullin and F. Laloë, Phys. Rev. A 82, 013618 (2010).

[24] M. C. Tichy, Entanglement and Interference of Identical Particles, $\mathrm{PhD}$ thesis, Universität Freiburg, www.freidok.uni-freiburg.de/volltexte/8233/, 2011.

[25] G. Cennini, C. Geckeler, G. Ritt, and M. Weitz, Phys. Rev. A 72, 051601 (2005).

[26] Z. Hadzibabic, S. Stock, B. Battelier, V. Bretin, and J. Dalibard, Phys. Rev. Lett. 93, 180403 (2004).

[27] K. F. Lee and J. E. Thomas, Phys. Rev. Lett. 88, 097902 (2002).

[28] K. Mayer, Many-particle quantum walks, Master's thesis, Universität Freiburg, www.freidok.unifreiburg.de/volltexte/8496/, 2011.

[29] M. C. Tichy, M. Tiersch, F. de Melo, F. Mintert, and A. Buchleitner, Phys. Rev. Lett. 104, 220405 (2010).

[30] C. K. Hong, Z. Y. Ou, and L. Mandel, Phys. Rev. Lett. 59, 2044 (1987).

[31] M. C. Tichy, H.-T. Lim, Y.-S. Ra, F. Mintert, Y.-H. Kim, and A. Buchleitner, Phys. Rev. A 83, 062111 (2011).

[32] Y.-S. Ra, M. C. Tichy, H.-T. Lim, O. Kwon, F. Mintert, and A. Buchleitner, Proc. Natl. Acad. Sci. USA 110, 1227 (2013).

[33] Y.-S. Ra, M. C. Tichy, H.-T. Lim, O. Kwon, F. Mintert, A. Buchleitner, and Y.-H. Kim, Nat. Commun. 4 (2013).

[34] M. Tillmann, S.-H. Tan, S. E. Stoeckl, B. C. Sanders, H. de Guise, R. Heilmann, S. Nolte, A. Szameit, P. Walther, BosonSampling with Controllable Distinguishability, arXiv:1403.3433, 2013.

[35] M. Reck, A. Zeilinger, H. J. Bernstein, P. Bertani, Phys. Rev. Lett. 73, 58 (1994). 
[36] N. Spagnolo, C. Vitelli, L. Aparo, P. Mataloni, F. Sciarrino, A. Crespi, R. Ramponi, and R. Osellame, Nat. Comm. 4, 1606 (2013).

[37] N. Spagnolo, L. Aparo, C. Vitelli, A. Crespi, R. Ramponi, R. Osellame, P. Mataloni, and F. Sciarrino, Sci. Rep. 2 (2012).

[38] T. Meany, M. Delanty, S. Gross, G. D. Marshall, M. J.
Steel, and M. J. Withford, Opt. Express 20, 26895 (2012).

[39] P. J. Shadbolt, M. R. Verde, A. Peruzzo, A. Politi, A. Laing, M. Lobino, J. C. F. Matthews, M. G. Thompson, and J. L. O'Brien, Nat. Photon. 6, 45 (2012).

[40] J. Bell, Physics 1, 195 (1964). 